\title{
ON OPTIMAL SENSOR LOCATIONS FOR NONPARAMETRIC IDENTIFICATION OF VISCOELASTIC MATERIALS
}

\author{
Agnes Runqvist*, Magnus Mossberg** and \\ Torsten Söderström* \\ ${ }^{*}$ Div. of Systems and Control, Dept. of Information \\ Technology, Uppsala University, Box 337, SE-75105 \\ Uppsala, Sweden. \\ E-mail: \{Agnes.Runqvist,Torsten.Soderstrom\}@it.uu.se \\ ** Dept. of Electrical Engineering, Karlstad University, \\ SE-651 88 Karlstad, Sweden. \\ E-mail: Magnus.Mossberg@kau.se
}

\begin{abstract}
The problem of optimal sensor locations in nonparametric identification of viscoelastic materials is considered. Different criteria of the covariance matrix, connected to A- and D-optimal experiment design, are considered and evaluated. The results of the paper can be used to design experiments with improved accuracy of the estimates.
\end{abstract}

Keywords: Experiment design, nonparametric identification, viscoelastic materials.

\section{INTRODUCTION}

In order to make efficient use of viscoelastic materials in constructions, it is important to have knowledge about the characteristics of the material. A viscoelastic material is characterized by its complex modulus that relates stress and strain, and can be determined through wave propagation experiments, as studied in (Sogabe and Tsuzuki, 1986), (Blanc, 1993) and (Hillström et al., 2000). To get good quality estimates, the collected data should contain as much valuable information as possible, and design parameters that influence the information content must thus be chosen carefully. Examples of such parameters are the input signal, the number of sensors used in the experiment, and the sensor locations. This paper is devoted to the design of optimal sensor locations for nonparametric identification of viscoelastic materials. The corresponding problem for the parametric case was considered in (Mossberg, 2004).
A solid theoretical base for optimal experiment design is built in (Fedorov, 1972) and (Pukelsheim, 1993), and is widely employed in different areas of electrical engineering. Some examples include sensor array signal processing (Hawkes and Nehorai, 1999), and robotics (Lizama and Šurdilović, 1996). In the control literature, a survey of the sensor location problem is presented in (Kubrusly and Malebranche, 1985).

\section{THE EXPERIMENT}

The behavior of a viscoelastic material is characterized by its complex modulus $E(\omega)$ that relates stress and strain of the material. The identification problem studied in this paper is the one of identifying the complex modulus of viscoelastic materials by the use of wave propagation methods. Fig. 1 shows the experimental setup where a bar specimen of length $L$ is axially impacted by a 

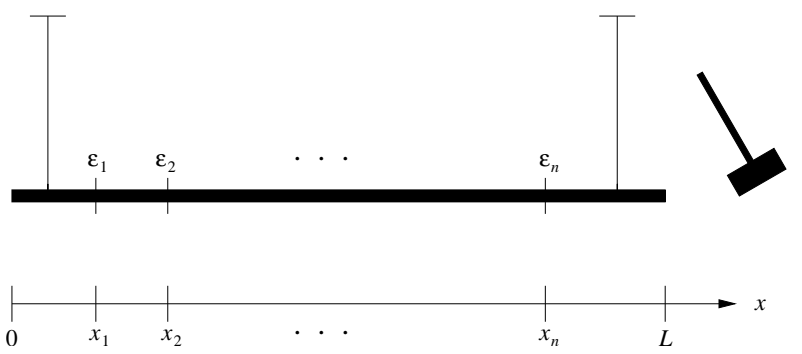

Fig. 1. Experimental setup

steel hammer or an air gun. As a result of the impact, longitudinal waves that travel back and forth along the bar, are generated. The associated strains $\varepsilon_{1}, \ldots, \varepsilon_{n}$ are measured at $n$ different sections located at $\boldsymbol{x}=\left[x_{1}, \ldots, x_{n}\right]^{T}$, at $N$ discrete time instances. Similar experiments can be designed for flexural waves by an impact perpendicular to the bar, as in (Mahata et al., 2003), and for torsional waves. This work will focus on the case with longitudinal waves but, can easily be expanded to cover flexural and torsional wave experiments.

\section{THE NONPARAMETRIC IDENTIFICATION}

By solving the wave equation in the frequency domain

$$
\frac{\partial^{2} \hat{\varepsilon}(x, \omega)}{\partial x^{2}}-\gamma^{2}(\omega) \hat{\varepsilon}(x, \omega)=0
$$

we get the expression (Hillström et al., 2000)

$$
\hat{\varepsilon}(x, \omega)=c_{1}(\omega) e^{\gamma(\omega) x}+c_{2}(\omega) e^{-\gamma(\omega) x},
$$

where $\hat{\varepsilon}(x, \omega)$ denotes the Fourier transform of the strain measurements at frequency $\omega$ and sensor location $x$. In $(2), c_{1}(\omega)$ and $c_{2}(\omega)$ are complex valued functions of $\omega$ which can be interpreted as amplitudes of waves at $x=0$, going in positive and negative direction, respectively. Here, $\gamma(\omega)$ is the wave propagation function, satisfying

$$
\gamma^{2}(\omega)=-\frac{\rho \omega^{2}}{E(\omega)} .
$$

By identifying $\gamma(\omega)$, the complex modulus $E(\omega)$ is easily determined through (3).

The strains caused by the impact on the bar are measured at $n$ sections located at $\boldsymbol{x}=$ $\left[x_{1}, \ldots, x_{n}\right]^{T}$. From (2), the system of equations

$$
\hat{\varepsilon}(\boldsymbol{x}, \omega)=\mathbf{A}(\boldsymbol{x}, \omega) \mathbf{c}(\omega),
$$

where

$$
\begin{gathered}
\mathbf{A}(\boldsymbol{x}, \omega)=\left[\begin{array}{cc}
\mathrm{e}^{\gamma(\omega) x_{1}} & \mathrm{e}^{-\gamma(\omega) x_{1}} \\
\vdots & \vdots \\
\mathrm{e}^{\gamma(\omega) x_{n}} & \mathrm{e}^{-\gamma(\omega) x_{n}}
\end{array}\right], \\
\mathbf{c}(\omega)=\left[c_{1}(\omega) c_{2}(\omega)\right]^{T}, \\
\hat{\boldsymbol{\varepsilon}}(\boldsymbol{x}, \omega)=\left[\hat{\varepsilon}\left(x_{1}, \omega\right), \ldots, \hat{\varepsilon}\left(x_{n}, \omega\right)\right]^{T},
\end{gathered}
$$

can be formed. An estimate of $\gamma(\omega)$ is obtained by minimizing the loss function

$$
U(\gamma(\omega), \mathbf{c}(\omega))=\|\hat{\boldsymbol{\varepsilon}}(\boldsymbol{x}, \omega)-\mathbf{A}(\boldsymbol{x}, \omega) \mathbf{c}(\omega)\|^{2}
$$

with respect to $\gamma(\omega)$ and $\mathbf{c}(\omega)$. Here, the unknowns $c_{1}(\omega)$ and $c_{2}(\omega)$ act as nuisance parameters, giving a total of three unknowns, and the number of sensors needed for identification must thus be $n \geq 3$. Alternatively, if there exist a free end at $x_{1}=0$, the boundary condition

$$
\hat{\varepsilon}_{1}\left(x_{1}, \omega\right)=0
$$

can be used as an extra strain measure, reducing the number of sensors needed by one. Once $\gamma(\omega)$ is determined, an estimate of the complex modulus at frequency $\omega$ can be obtained through (3).

In (Mahata et al., 2003), an expression for the covariance matrix for the estimate of the complex modulus $E(\omega)$ is derived, assuming the measurement noise to be white and the SNR large. For notational simplicity and to stress that the matrix $\mathbf{A}(\boldsymbol{x}, \omega)$ implicitly is an analytic function of the complex modulus, it is from here onwards replaced by $\mathbf{A}\left(\mathbf{e}_{\omega}\right)$, where

$$
\mathbf{e}_{\omega}=\left[e_{r}(\omega) e_{i}(\omega)\right]^{T},
$$

such that

$$
E(\omega)=e_{r}(\omega)+\mathrm{i} e_{i}(\omega) .
$$

The covariance matrix can in these notations be expressed as

$$
\mathrm{E}\left\{\left(\hat{\mathbf{e}}_{\omega_{l}}-\mathbf{e}_{\omega_{l} 0}\right)\left(\hat{\mathbf{e}}_{\omega_{k}}-\mathbf{e}_{\omega_{k} 0}\right)^{T}\right\}=\frac{\lambda}{h\left(\boldsymbol{x}, \omega_{l}\right)} \delta_{l, k} \mathbf{I}_{2},
$$

where $\lambda$ is the noise variance, $\delta_{l, k}$ the Kronecker delta function, and

$$
\omega_{k}= \begin{cases}\frac{2 \pi k}{N T}, & 0 \leq k \leq \frac{N}{2} \\ \frac{2 \pi(k-N)}{N T}, & \frac{N}{2} \leq k \leq N\end{cases}
$$

Further,

$$
\begin{aligned}
h(\boldsymbol{x}, \omega)= & 2 \hat{\varepsilon}^{*}(\boldsymbol{x}, \omega) \mathbf{A}^{\dagger *}\left(\mathbf{e}_{\omega 0}\right) \mathbf{A}_{r}^{*}\left(\mathbf{e}_{\omega 0}\right) \mathbf{P}\left(\mathbf{e}_{\omega 0}\right) \\
& \times \mathbf{A}_{r}\left(\mathbf{e}_{\omega 0}\right) \mathbf{A}^{\dagger}\left(\mathbf{e}_{\omega 0}\right) \hat{\boldsymbol{\varepsilon}}(\boldsymbol{x}, \omega) \\
= & 2 \mathbf{c}^{*}(\omega) \mathbf{A}_{r}^{*}\left(\mathbf{e}_{\omega 0}\right) \mathbf{P}\left(\mathbf{e}_{\omega 0}\right) \mathbf{A}_{r}\left(\mathbf{e}_{\omega 0}\right) \mathbf{c}(\omega),
\end{aligned}
$$

where $\mathbf{A}^{\dagger}\left(\mathbf{e}_{\omega}\right)$ is the pseudo-inverse of $\mathbf{A}\left(\mathbf{e}_{\omega}\right)$, $\mathbf{P}\left(\mathbf{e}_{\omega}\right)$ the orthogonal projection onto the null space of $\mathbf{A}^{*}\left(\mathbf{e}_{\omega}\right)$, and $\mathbf{A}_{r}\left(\mathbf{e}_{\omega}\right)$ is defined as

$$
\mathbf{A}_{r}\left(\mathbf{e}_{\omega}\right)=\frac{\partial \mathbf{A}\left(\mathbf{e}_{\omega}\right)}{\partial e_{r}(\omega)} .
$$

From (12) we see that the estimate of the complex modulus at a particular frequency is uncorrelated with the estimate at any other frequency. We also see that the estimates of the real and the imaginary part of $E(\omega)$ are uncorrelated at any given frequency, and that the variances of these 
estimates are equal and proportional to $1 / h(\omega)$. Another important aspect of the covariance matrix is that it is implicitly dependent on the sensor locations $\boldsymbol{x}=\left[x_{1}, \ldots, x_{n}\right]^{T}$ through $\mathbf{A}\left(\mathbf{e}_{\omega}\right)$, as can be seen from equations (5), (14) and (15). The variance of the estimates is thus influenced by the choice of sensor locations and the number of sensors $n$, which in turn means that an optimal choice of $\boldsymbol{x}$ will improve the accuracy of the estimates.

\section{OPTIMAL EXPERIMENT DESIGN}

In nonparametric identification, an estimate of the complex modulus $E(\omega)$ is calculated for every frequency of interest. As a result, the covariance matrix of the estimates must be evaluated separately at every such frequency. Naturally, it is desirable to keep the variance to a minimum at every given frequency, a task not necessarily trivial. As it is not possible to consider each frequency individually in the optimization process, a pertinent scalar measure of the performance is needed. In this paper, the three scalar criteria

$$
\begin{aligned}
V_{1}(\boldsymbol{x}) & =\frac{1}{h\left(\boldsymbol{x}, \omega_{k}\right)}, \\
V_{2}(\boldsymbol{x}) & =\sum_{k} \frac{1}{h^{2}\left(\boldsymbol{x}, \omega_{k}\right)}, \\
V_{3}(\boldsymbol{x}) & =\sum_{k} \frac{1}{h\left(\boldsymbol{x}, \omega_{k}\right)},
\end{aligned}
$$

are considered and evaluated. Here, $V_{1}(\boldsymbol{x})$ will minimize the covariance function at a particular frequency, while $V_{2}(\boldsymbol{x})$ and $V_{3}(\boldsymbol{x})$ by simple mathematical relationships are equivalent to minimizing, respectively, the mean of the determinant or
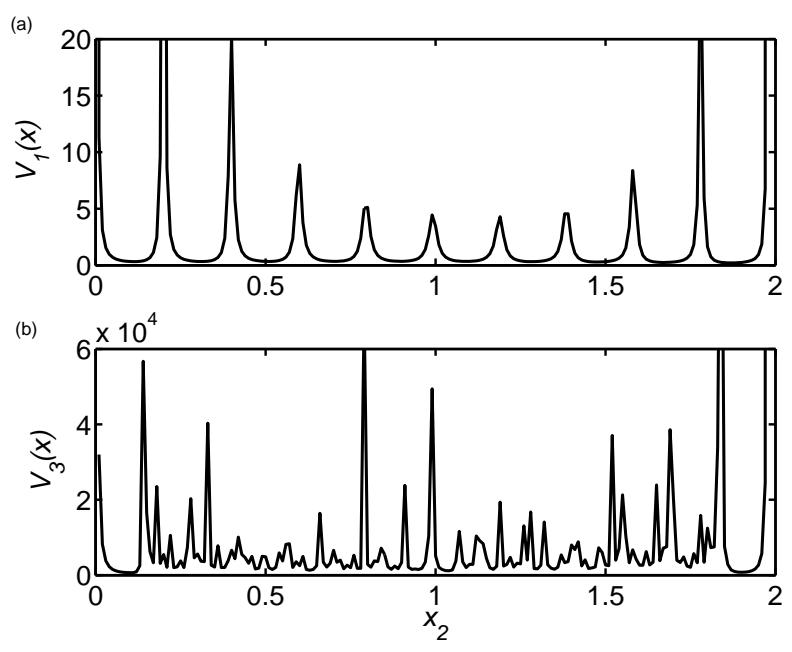

Fig. 2. For $n=3$ sensors, criterion $V_{1}(\boldsymbol{x})$ (a) and criterion $V_{3}(\boldsymbol{x})$ (b) are shown as functions of sensor location $x_{2}$. Sensors $x_{1}$ and $x_{3}$ are locked to position 0 and 1.98, respectively. The behavior of criterion $V_{2}(\boldsymbol{x})$ is similar to that of $V_{3}(\boldsymbol{x})$ and is thus omitted. the mean of the trace of the covariance matrix, over some frequency range. This frequency range can be chosen to cover an interval of particular interest, and will comprise all the discrete frequency points, within that interval, at which the complex modulus are calculated. The argument $\boldsymbol{x}$ of the criteria will be omitted from here onwards.

In the the theory of optimal experiment design, treated in (Fedorov, 1972) and (Pukelsheim, 1993) among others, the criteria $V_{1}$ and $V_{3}$ implies Aoptimality, since A-optimality is based on the average variance of the parameter estimates, i.e. the trace of the covariance matrix. In the same way, the criterion $V_{2}$ implies D-optimality, which is based on minimizing the determinant of the covariance matrix. D-optimality is the most common criterion used in optimal design, since it it is invariant under re-parameterizations and guarantees that the confidence ellipsoid is kept small.

\section{OPTIMIZATION ASPECTS}

The three criteria presented in (16)-(18) involves very hard optimization problems with several local minima separated by high peaks, as can be seen in Fig. 2. It is clear that a global optimization algorithm is needed, and in this study an algorithm based on multilevel coordinate search, presented in (Huyer and Neumaier, 1999), was used. Each of these criteria was minimized with respect to the sensor positions for different number of sensors $n$. For implementation aspects, and in accordance with the work in (Mossberg, 2004), the following constraints were imposed on the optimization:

$$
\begin{gathered}
0+\delta_{1} \leq x_{i} \leq L-\delta_{2}, \quad i=1, \ldots, n \\
x_{i+1}-x_{i} \geq \delta_{3}, \quad i=1, \ldots, n-1
\end{gathered}
$$

Here, $\delta_{1}$ is the minimum distance between a sensor and the left end of the bar, $\delta_{2}$ the minimum distance between a sensor and the point of excitation at the right end of the bar, and $\delta_{3}$ the minimum distance between two sensors. The numerical values chosen in this study were $\delta_{1}=0$ and $\delta_{2}=\delta_{3}=0.02 \mathrm{~m}$.

This work is based on the experimental data obtained in (Hillström et al., 2000), where the material PMMA (plexiglass) was studied. A rod of $L=2 \mathrm{~m}$ was axially impacted, and strain data collected at $N=4096$ discrete time instances, with a sampling interval of $20 \mu \mathrm{s}$. In the expression for the covariance matrix in (12) and (14), the true complex modulus at each frequency is needed. These values are here replaced by the estimated complex modulus obtained through (3), where $\gamma(\omega)$ is the mean of 10 identification experiments, see (8). In (Hillström et al., 2000) two approaches to sensor configuration were evaluated. In one 


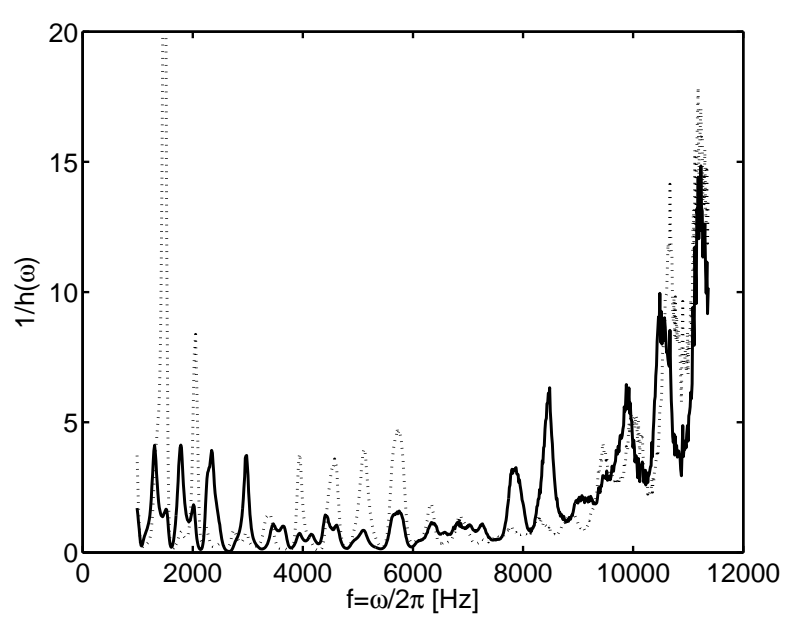

Fig. 3. $1 / h(\boldsymbol{x}, \omega)$ when criterion $V_{1}$ was minimized for 5 sensors at approximately $3 \mathrm{kHz}$ (dotted), and $1 / h(\boldsymbol{x}, \omega)$ for reference sensor configuration $\boldsymbol{x}_{\text {ref }}$ (solid), as a function of frequency.

approach the sensors were spaced uniformly on the rod, so that the distance between any two sensors was a multiple of $0.2 \mathrm{~m}$. This configuration was found to have some apparent drawbacks, as the estimate can be expected to be inaccurate if all sensors are placed so that the distance between any two sensors, or the distance between the free left end and the middle sensor if only three sensors are used, is an integral multiple of half a wavelength of the frequency, at which the identification is carried out. In the other approach, five sensors were unevenly placed on the bar in order to minimize the risk for such critical conditions to occur. This configuration, $\boldsymbol{x}_{\text {ref }}=$ $[0,0.290,0.646,1.078,1.600]^{T}$, has proven to give a low variance for all frequencies considered in the identification experiment, and has thus become a standard configuration in subsequent work. It is also the configuration used when collecting the data used in this study, and will be referred to as the 'reference configuration' in what follows. The critical conditions discussed above are important to keep in mind during the optimizations.

\section{INVESTIGATIONS}

The way the optimization problem is formulated allows us to pose a number of questions concerning the sensor locations and the design of the experiment. Some of these questions will be further discussed in the examples in this section.

Example 1. Is $V_{1}$ a good criterion to use? What happens at other frequencies than $\omega_{k}$, the frequency of minimization?

During the optimizations it was clear that the surface produced by the criterion $V_{1}$ is particularly hard for optimization purposes, and the

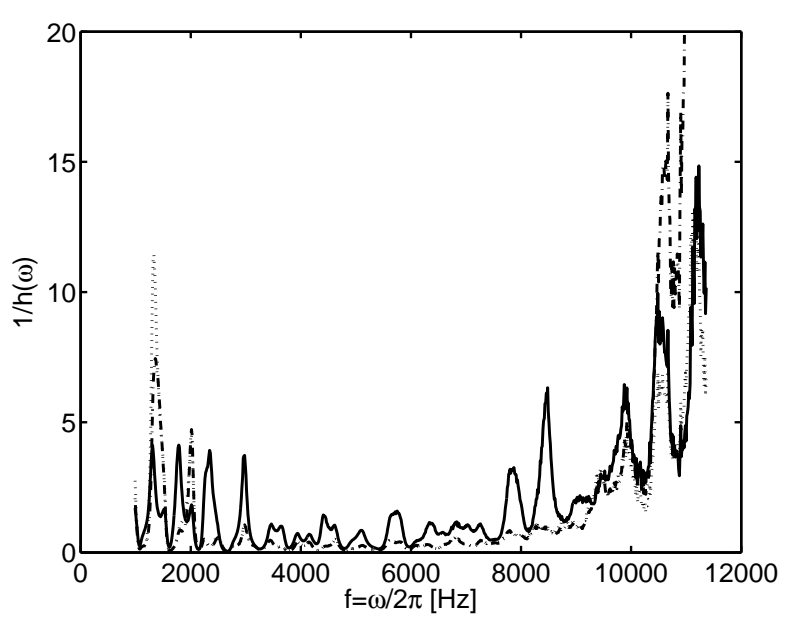

Fig. 4. $1 / h(\boldsymbol{x}, \omega)$ against frequency for $n=5$ sensors for reference sensor placement $\boldsymbol{x}_{r e f}$ in (5) (solid), optimal sensor placement by $V_{2}$ (dash-dotted), and $V_{3}$ (dotted). $V_{2}$ and $V_{3}$ were minimized in the interval 2 to $8 \mathrm{kHz}$.

optimization algorithm did in many cases not converge to the global minimum for this criterion. Furthermore, minimizing the variance at a single frequency showed a strong tendency to press up the variance at other frequencies, as can be seen from Fig. 3. The criteria $V_{2}$ and $V_{3}$ proved to be simpler for optimization purposes, even though summation over a range of frequencies significantly increases the computational load. Another strength of these criteria is that the designer of the experiment can choose an interval of frequencies, in which the variance of the estimates should be kept low. Similarly to $V_{1}$, both $V_{2}$ and $V_{3}$ experience the problem of large inaccuracy in the estimates for frequencies outside the range of minimization, but within the chosen interval the variance is significantly lower than for the

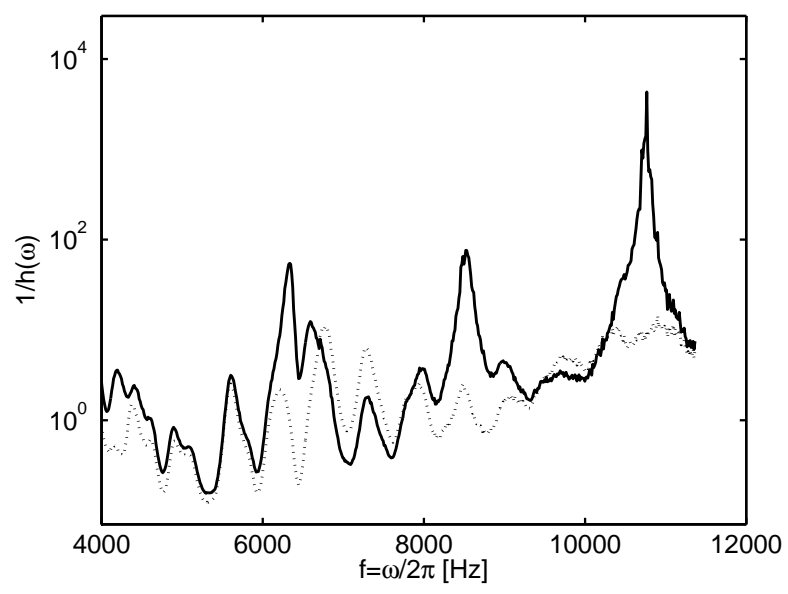

Fig. 5. $1 / h(\boldsymbol{x}, \omega)$ against frequency for criterion $V_{1}$, minimized at approximately $5.2 \mathrm{kHz}$. Optimal sensor placement for $n=3$ sensors (critical sensor configuration for $6.4 \mathrm{kHz}, 8.5$ $\mathrm{kHz}$, and $11 \mathrm{kHz}$ ), (solid), and for $n=4$ sensors, (dotted). Observe the logarithmic scale. 
Table 1. Minimum values of $V_{1}, V_{2}$ and $V_{3}$ and corresponding $\boldsymbol{x}$ for different number of sensors $n$. $V_{1}$ minimized at $3 \mathrm{kHz}$, and $V_{2}$ and $V_{3}$ on the interval $I=2-8 \mathrm{kHz}$.

\begin{tabular}{|c|c|c|c|c|c|}
\hline & $n$ & $\boldsymbol{x}^{T}$ & $V_{i}(\boldsymbol{x})$ & $\begin{array}{c}\text { Average } 1 / h(\boldsymbol{x}, \omega) \\
\text { on interval } I\end{array}$ & $\begin{array}{c}\text { Maximal } 1 / h(\boldsymbol{x}, \omega) \\
\text { on interval } I\end{array}$ \\
\hline \multirow{6}{*}{$V_{1}$} & 3 & {$\left[\begin{array}{llll}0 & 1.7238 & 1.8365\end{array}\right]$} & 1.339 & 1.049 & 8.846 \\
\hline & 4 & {$\left[\begin{array}{lllll}0 & 0.0200 & 1.7364 & 1.8549\end{array}\right]$} & 0.993 & 0.678 & 4.444 \\
\hline & 5 & {$\left[\begin{array}{lllllll}0 & 0.0200 & 0.2056 & 1.7794 & 1.7994\end{array}\right]$} & 0.736 & 0.990 & 4.798 \\
\hline & 6 & {$\left[\begin{array}{lllllll}0 & 0.0200 & 0.0400 & 0.1973 & 1.7889 & 1.8089\end{array}\right]$} & 0.630 & 0.689 & 4.860 \\
\hline & 7 & {$\left[\begin{array}{llllllll}0 & 0.0400 & 0.0600 & 0.5732 & 1.7886 & 1.8086 & 1.8286\end{array}\right]$} & 0.547 & 0.429 & 1.661 \\
\hline & $\boldsymbol{x}_{r e f}$ & {$\left[\begin{array}{llllll}0 & 0.2900 & 0.6460 & 1.0780 & 1.6000\end{array}\right]$} & 3.729 & 0.898 & 3.921 \\
\hline \multirow{6}{*}{$V_{2}$} & 3 & {$\left[\begin{array}{llll}0.0011 & 1.8449 & 1.9264\end{array}\right]$} & 363.592 & 0.652 & 2.939 \\
\hline & 4 & {$\left[\begin{array}{lllll}0 & 0.0724 & 1.8073 & 1.9113\end{array}\right]$} & 101.586 & 0.392 & 1.219 \\
\hline & 5 & {$\left[\begin{array}{lllll}0 & 0.0923 & 0.1861 & 1.8093 & 1.9063\end{array}\right]$} & 63.364 & 0.313 & 1.063 \\
\hline & 6 & 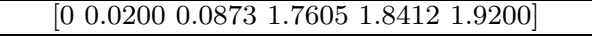 & 42.552 & 0.252 & 0.812 \\
\hline & 7 & 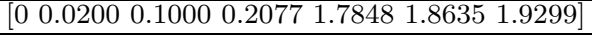 & 29.979 & 0.213 & 0.729 \\
\hline & $\boldsymbol{x}_{r e f}$ & {$\left[\begin{array}{llllll}0 & 0.2900 & 0.6460 & 1.0780 & 1.6000\end{array}\right]$} & 690.143 & 0.898 & 3.921 \\
\hline \multirow{6}{*}{$V_{3}$} & 3 & {$\left[\begin{array}{llll}0.0005 & 1.8399 & 1.9247\end{array}\right]$} & 318.707 & 0.649 & 3.083 \\
\hline & 4 & {$\left[\begin{array}{lllll}0 & 0.0732 & 1.8145 & 1.9187\end{array}\right]$} & 192.141 & 0.391 & 1.278 \\
\hline & 5 & {$\left[\begin{array}{lllll}0 & 0.0200 & 0.0908 & 1.8123 & 1.9149\end{array}\right]$} & 152.680 & 0.311 & 1.006 \\
\hline & 6 & {$\left[\begin{array}{lllllll}0 & 0.0200 & 0.0900 & 1.7949 & 1.8941 & 1.9800\end{array}\right]$} & 121.115 & 0.247 & 0.869 \\
\hline & 7 & {$\left[\begin{array}{lllllll}0 & 0.0200 & 0.1000 & 0.2084 & 1.8012 & 1.8955 & 1.9800\end{array}\right]$} & 104.421 & 0.213 & 0.805 \\
\hline & $\boldsymbol{x}_{r e f}$ & {$\left[\begin{array}{llllll}0 & 0.2900 & 0.6460 & 1.0780 & 1.6000\end{array}\right]$} & 440.924 & 0.898 & 3.921 \\
\hline
\end{tabular}

reference sensor configuration $\boldsymbol{x}_{r e f}$, see Fig. 4. It can also be seen that minimizing the criteria $V_{2}$ and $V_{3}$ produce a similar result. Within the chosen interval, neither of these two criteria tolerate large inaccuracy in the estimates due to the critical conditions discussed in Section 5. In criterion $V_{1}$ however, only one single frequency is considered and bad behavior at other frequencies is thus allowed. The use of more than three sensors significantly reduces this problem, as the possibility of a critical sensor configurations decreases, see Fig. 5. This should be true for all criteria used.

Example 2. What is the potential to improved performance compared to the standard configuration $\boldsymbol{x}_{\text {ref }}$ ?

In Table 1 some numerical values for $\boldsymbol{x}_{r e f}$ are displayed together with the corresponding results for the three criteria considered in this study. From the table we see that already with three optimally placed sensors there is a significant decrease of the function values, compared to the reference configuration. For the same number of sensors in the optimal configuration as in the reference, the decrease of function values is even greater. In Table 1 we see that for $V_{1}$, the function value has gone from 3.729 to 0.736 , corresponding to a decrease of the variance of the estimates at the frequency of minimization by $80 \%$. For criterion $V_{2}$ (and $V_{3}$ ) the related decrease of functional values corresponds to a decrease of $65 \%$ (65\%) of the average variance within the interval of minimization or a decrease of $73 \%(74 \%)$ of the maximal variance within that interval. These results indicate that the quality of the estimate of the real and imaginary part of $E(\omega)$ can be greatly improved, at one single frequency or within a range of frequencies, if an optimal sensor configuration is used.
Example 3. What is a good number of sensors to use?

In Table 1 there is a continuous decrease of function values and related variances with an increasing number of sensors. The gain from adding one more sensor is however reduced as more sensors are added, leaving the designer with the choice of how many sensors it is reasonable to use. It is apparent from the table that a great improvement is achieved for the criteria $V_{2}$ and $V_{3}$ when increasing the number of sensors from three to four, but that more than four sensors give a more moderate improvement. This can be compared with the optimal experimental design for the parametric case studied in (Mossberg, 2004), where a great improvement was noticed for up to five sensors. For the criterion $V_{1}$, there is no such great leap in function values. One reason for this could be that these values only depend on the behavior at one single frequency point and that three sensors are enough to achieve a reasonably good value at that point, although using more sensors will improve it further. As discussed above, it is also preferable to use more than three sensors to avoid a situation where the estimation algorithm breaks down for some frequencies.

Example4. What is the effect of constraining the sensors to different positions?

The results in Table 1 were all achieved by implementing the constraints (19) and (20). If the optimal sensor locations are studied, it is apparent that for all three criteria, a number of sensors tend to group as close together as constraint (20) allows, close to the left end of the bar. For criterion $V_{1}$, the same behavior is also true for the right end of the bar. We therefore also examined the optimization problem without the constraint (20), i.e. 
Table 2. Minimum values of $V_{1}$ and $V_{2}$ with corresponding $\boldsymbol{x}$ for different number of sensors $n$. $V_{1}$ minimized at $3 \mathrm{kHz}$, and $V_{2}$ on the interval $I=2-8 \mathrm{kHz}$.

\begin{tabular}{|c|c|c|c|c|c|}
\hline & $n$ & $\boldsymbol{x}^{T}$ & $V_{i}(\boldsymbol{x})$ & $\begin{array}{c}\text { Average } 1 / h(\boldsymbol{x}, \omega) \\
\text { on interval } I\end{array}$ & $\begin{array}{c}\text { Maximal } 1 / h(\boldsymbol{x}, \omega) \\
\text { on interval } I\end{array}$ \\
\hline \multirow{5}{*}{$V_{1}$} & 3 & {$\left[\begin{array}{llll}0 & 1.7238 & 1.8365\end{array}\right]$} & 1.339 & 1.049 & 8.846 \\
\hline & 4 & {$\left[\begin{array}{lllll}0 & 0 & 1.7249 & 1.8373\end{array}\right]$} & 0.987 & 0.937 & 8.350 \\
\hline & 5 & {$\left[\begin{array}{lllll}0 & 0 & 0.1436 & 1.7979 & 1.7979\end{array}\right]$} & 0.722 & 12.523 & 307.831 \\
\hline & 6 & {$\left[\begin{array}{lllllll}0 & 0 & 0 & 0.1668 & 1.7907 & 1.7907\end{array}\right]$} & 0.611 & 4.630 & 55.752 \\
\hline & 7 & {$\left[\begin{array}{llllllll}0 & 0 & 0 & 0.5304 & 1.7889 & 1.7889 & 1.7889\end{array}\right]$} & 0.502 & 2.056 & 33.839 \\
\hline \multirow{5}{*}{$V_{2}$} & 3 & {$\left[\begin{array}{llll}0.0011 & 1.8449 & 1.9264\end{array}\right]$} & 363.3592 & 0.652 & 2.939 \\
\hline & 4 & {$\left[\begin{array}{lllll}0 & 0.0724 & 1.8073 & 1.9113\end{array}\right]$} & 101.586 & 0.392 & 1.219 \\
\hline & 5 & {$\left[\begin{array}{llllll}0 & 0 & 0.0756 & 1.8027 & 1.9062\end{array}\right]$} & 63.175 & 0.310 & 1.030 \\
\hline & 6 & {$\left[\begin{array}{llllll}0 & 0 & 0.0728 & 1.7670 & 1.8558 & 1.9348\end{array}\right]$} & 40.747 & 0.244 & 0.778 \\
\hline & 7 & {$\left[\begin{array}{llllllll}0 & 0 & 0.0520 & 0.0935 & 1.7747 & 1.8629 & 1.9360\end{array}\right]$} & 29.729 & 0.212 & 0.710 \\
\hline
\end{tabular}

Table 3. Minimum values of $V_{1}, V_{2}$ and $V_{3}$ and corresponding $\boldsymbol{x}$ for different number of sensors $n$. $V_{1}$ minimized at $3 \mathrm{kHz}$, and $V_{2}$ and $V_{3}$ on the interval $I=2-8 \mathrm{kHz}$.

\begin{tabular}{|c|c|c|c|c|c|}
\hline & $n$ & $\boldsymbol{x}^{T}$ & $V_{i}(\boldsymbol{x})$ & $\begin{array}{c}\text { Average } 1 / h(\boldsymbol{x}, \omega) \\
\text { on interval } I\end{array}$ & $\begin{array}{c}\text { Maximal } 1 / h(\boldsymbol{x}, \omega) \\
\text { on interval } I\end{array}$ \\
\hline \multirow{5}{*}{$V_{1}$} & 3 & {$\left[\begin{array}{llll}0 & 1.7238 & 1.8365\end{array}\right]$} & 1.339 & 1.049 & 8.846 \\
\hline & 4 & {$\left[\begin{array}{llll}0 & 0.4771 & 1.7968 & 1.8168\end{array}\right]$} & 1.041 & 1.293 & 7.372 \\
\hline & 5 & {$\left[\begin{array}{lllll}0 & 0.1500 & 0.3323 & 1.7830 & 1.7030\end{array}\right]$} & 0.848 & 0.791 & 4.252 \\
\hline & 6 & {$\left[\begin{array}{llllll}0 & 0.3133 & 0.4585 & 1.7700 & 1.7900 & 1.8100\end{array}\right]$} & 0.721 & 0.551 & 1.938 \\
\hline & 7 & 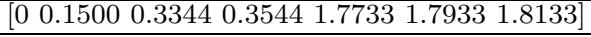 & 0.632 & 0.434 & 1.784 \\
\hline \multirow{5}{*}{$V_{2}$} & 3 & {$\left[\begin{array}{llll} & 1.7463 & 1.8242\end{array}\right]$} & 399.083 & 0.716 & 2.843 \\
\hline & 4 & {$\left[\begin{array}{llll}0 & 0.2133 & 1.7642 & 1.8500\end{array}\right]$} & 128.327 & 0.443 & 1.314 \\
\hline & 5 & {$\left[\begin{array}{lllll}0 & 0.2308 & 0.3291 & 1.7633 & 1.8500\end{array}\right]$} & 84.591 & 0.361 & 1.057 \\
\hline & 6 & {$\left[\begin{array}{llllll}0 & 0.2326 & 0.3254 & 1.7317 & 1.7897 & 1.8500\end{array}\right]$} & 61.245 & 0.303 & 0.869 \\
\hline & 7 & {$\left[\begin{array}{lllllll}0 & 0.1500 & 0.2336 & 0.3422 & 1.7410 & 1.7953 & 1.8500\end{array}\right]$} & 44.769 & 0.263 & 0.717 \\
\hline
\end{tabular}

$\delta_{3}=0$. This gave the results displayed in Table 2 . It can be seen that for all three criteria, more sensors are placed at the left end of the bar $\left(x_{i}=0\right)$ as the number of sensors increase, giving slightly lower values than those in Table 1. This indicates that, instead of having an increasing number of sensors, higher accuracy in the estimates can be achieved by using a more accurate sensor at this measurement point. As the strain measures at this point are obtained by the boundary condition (9), this is only of theoretical interest. For criteria $V_{1}$, sensors also group together towards the right end of the bar, again indicating that three sensors are enough to get a good accuracy at one single frequency point.

Example 5. What effect will a tightening of the constraints have?

In a regular experiment, the sensors can not be placed within 10 diameters from the ends of the bar, due to transient effects in the reflected waves. In order to get an optimal sensor configuration suitable for an experiment, we therefore repeated the optimizations considering these limitations for bars up to $0.015 \mathrm{~m}$ of diameter, i.e. $\delta_{1}=\delta_{2}=0.15$ $\mathrm{m}$ and $\delta_{3}=0.02 \mathrm{~m}$. As the previous results in this study show that it is desirable to utilize the boundary condition (9), one sensor was here fixed in $x_{1}=0$ in order to simplify the optimization. The result is displayed in Table 3. As it is no longer possible to place sensors as close to the ends as in Table 1 and Table 2, some changes in the sensor configurations can be noted, giving slightly higher function values. The general behavior is however in agreement with the earlier results.

Example6. Do the optimal sensor configurations show any characteristic pattern?

From the examples above, and in accordance with the findings in the parametric case (Mossberg, 2004), it is obvious that it is advantageous to place sensors close to the point of excitation at the right end of the bar, and close to the left end. Furthermore, it is advantageous to make use of the information available through the boundary condition (9). This behavior is true for all three criteria.

\section{CONCLUSIONS}

This paper treats the problem of optimal sensor locations for estimating the complex modulus of a viscoelastic material, using longitudinal wave propagation experiments. The procedure used in this study also applies to the more general case of identification experiments, where the covariance matrix is dependent on the sensor locations. The number of sensors in the experiment, and their positions, affect the variance of the estimates, and results from this study can thus be used to increase the quality of the estimates in future experiments. The three design criteria $V_{1}, V_{2}$ and $V_{3}$ in (16)-(18) were studied. The criterion $V_{1}$ 
minimizes the variance at one single frequency point, while the criteria $V_{2}$ and $V_{3}$ minimize the variance over an interval of frequencies, based on the trace and the determinant of the covariance matrix, respectively. It was found that minimizing the variance at a limited number of frequencies leads to increased variances at frequencies outside the interval of minimization. This is particularly bad for $V_{1}$, while $V_{2}$ and $V_{3}$ are less sensitive as the designer can choose an interval of frequencies, in which the variance should be kept low. The criteria $V_{2}$ and $V_{3}$ were also found to have a similar behavior, both in actual sensor placements and in the behavior inside the interval of minimization. Furthermore, more than three sensors should be used. Adding more sensors will continously decrease the variances of the estimates, and it is up to the designer to decide when the cost of adding one more sensor is not compensated by the gain. Finally, it is advantageous to place sensors near the ends of the bar. Moreover, placing more accurate sensors at critical points of the bar was found to have an effect similar to that of increasing the number of sensors.

There are of course other experimental aspects that influence the accuracy of the estimates. One example is the input signal to the identification experiment, and this is a topic for future work.

\section{REFERENCES}

Blanc, R. H. (1993). Transient wave propagation methods for determining the viscoelastic properties of solids. Journal of Applied Mechanics 60, 763-768.

Fedorov, V. V. (1972). Theory of Optimal Experiments. Academic Press.

Hawkes, M. and A. Nehorai (1999). Effects of sensor placement on acoustic vector-sensor array performance. IEEE Journal of Oceanic Engineering 24(1), 33-40.

Hillström, L., M. Mossberg and B. Lundberg (2000). Identification of complex modulus from measured strains on an axially impacted bar using least squares. Journal of Sound and Vibration 230(3), 689-707.

Huyer, W. and A. Neumaier (1999). Global optimization by multilever coordinate search. Journal of Global Optimization 14(4), 331355.

Kubrusly, C. S. and H. Malebranche (1985). Sensors and controllers location in distributed systems - a survey. Automatica 21(2), 117128.

Lizama, E. and D. Šurdilović (1996). Designing G-optimal experiments for robot dynamics identification. In: Proc. Int. Conf. on Robotics and Automation. Minneapolis, MN, USA. pp. 311-316.

Mahata, K., S. Mousavi, T. Söderström, M. Mossberg, U. Valdek and L. Hillström (2003). On the use of flexural wave propagation experiments for identification of complex modulus. IEEE Trans. on Control Systems Technology 11(6), 863-874.

Mossberg, M. (2004). Optimal experimental design for identification of viscoelastic materials. IEEE Trans. on Control Systems Technology 12(4), 578-582.

Pukelsheim, F. (1993). Optimal Design of Experiments. Wiley.

Sogabe, Y. and M. Tsuzuki (1986). Identification of the dynamic properties of linear viscoelastic materials by wave prpagation testing. Bulletin of the Japan Society of Mechanical Engineers 29(254), 2410-2417. 